\title{
Solitary pulmonary nodules: what to do when the resection margin is too close
}

\author{
Daniel P. Dolan", Scott J. Swanson" \\ Division of Thoracic Surgery, Department of Surgery, Brigham and Women's Hospital Boston, MA, USA \\ "Both authors contributed equally to this work. \\ Correspondence to: Daniel Dolan, MD. Division of Thoracic Surgery, Department of Surgery, Brigham and Women's Hospital (BWH)/Harvard \\ Medical School (HMS), 75 Francis Street, Boston, MA, 02115, USA. Email: ddolan7@bwh.harvard.edu.
}

Received: 27 May 2020; Accepted: 21 June 2020; Published: 25 August 2021.

doi: $10.21037 /$ ccts-20-107

View this article at: http://dx.doi.org/10.21037/ccts-20-107

\section{Introduction}

Determination of the nature of solitary pulmonary nodules (SPNs) remains a significant challenge. SPNs are rounded opacities, well or poorly de-fined, measuring up to $3 \mathrm{~cm}$ in diameter (1). These lesions are discovered either incidentally due to increased use of imaging technology or on CT lung screening (2). Multiple methods of determining SPN histology can be done but surgery remains the mainstay of diagnosis and treatment for lesions at high risk of being non-small cell lung cancer (NSCLC) (3).

The National Comprehensive Cancer Network (NCCN) Guidelines recommend "margins of $2 \mathrm{~cm}$ or the size of the nodule" for segmentectomy and wedge resection of NSCLC (4). Margins under this size are considered close. These recommendations were informed by the results of Sawabata et al., Wolf et al., Takahashi et al., and Mohiuddin et al. (5-8). Sawabata et al., reported on 118 NSCLC sublobar resections and found that with margin distance over $2 \mathrm{~cm}$ and margin distance greater than the maximum tumor diameter that no malignant cells were identified at the margin edge when cytopathologically examined (5). Wolf et al. followed 138 patients who underwent sublobar resection for Stage I NSCLC $\leq 2 \mathrm{~cm}$ and, over an average of 49.6 months, found that margin distance over $9 \mathrm{~mm}$ had the greatest increase in locoregional-recurrence free survival and a margin distance over $11 \mathrm{~mm}$ had the longest overall survival (6). Mohiuddin et al. expanded on this work for NSCLC $\leq 2 \mathrm{~cm}$ by examining 479 patients to 2 years after sublobar resection (8). They found that a margin distance of $\geq 1.5 \mathrm{~cm}$ significantly decreased the local recurrence risk. Lastly, Takahashi et al. examined 32 patients with a median tumor size of $2.0 \mathrm{~cm}$ and showed that in 8 of their 9 recurrences, at a median follow up of 39 months, the margin distance/tumor size ratio was $<1$ (7).

Compliance with the NCCN guidelines can be difficult to achieve in practice due to location or other complexities arising during the operation with the resulting tumor margin being quite small. The discovery that the margin is close can come either intraoperatively, on examination after specimen removal from the body, or postoperatively several days later when the patient has been potentially already been discharged from the hospital. If the lesion proves benign, then a close margin is acceptable. However, a close margin in a lesion that is determined to be NSCLC is much more concerning. Yet, how to deal with this problem is also dependent on when the close margin is found, the location of the tumor relative to the hilum (central $v s$. peripheral), which lobe the tumor is in, and the patient's medical status.

\section{General principles}

If the margin is found to be inadequate intraoperatively then efforts should be made to obtain a better margin before leaving the operating room (OR). Exceptions to this would include if the patient is decompensating or if additional resection would significantly compromise lung function postoperatively. If the margin is found to be close on final pathology the patient's options need to be carefully considered. The NCCN guidelines state that for Stage IA to IIB NSCLC if a positive margin is found at time of surgery then reresection should be performed (4). If the 
positive margin is identified after the original operation in patients with good lung function and a peripheral lesion, then return to the OR for additional sublobar resection can be done with the awareness that the chances of completion lobectomy are much higher depending on the duration from the initial operation due to adhesions that develop postoperatively. For completion resections of central lesions, great care will be necessary during surgery as fibrosis and scarring of hilar structures is almost certain to be present due to lymph node sampling at initial surgery and general healing in the area.

The decision to return to the OR for a close margin is much more perilous for patients with poor lung function. Typically, these patients have other comorbidities that make extensive surgery prohibitive initially. Multidisciplinary discussions with medical oncology and radiation oncology are advised to determine the best course for the patient if guideline concordant care is not possible. Patients may be monitored more closely with serial imaging, undergo SBRT, or chemotherapy as potential ways to manage the situation $(4,9,10)$.

In the remainder of this paper we will discuss our recommendations for various scenarios after a sublobar resection with a close margin. Peripheral is defined as outer half of the lung and central is defined as the inner half of the lung.

\section{Right upper lobe sublobar resection with close margin}

The right upper lobe consists of three bronchopulmonary segments; apical, posterior, and anterior. If the malignant SPN that was resected was located peripherally and a close margin was found intraoperatively, it is usually possible to resect additional tissue to create a more proximal staple line for patients with good preoperative lung function. Similarly, additional resection at a later date can usually be accomplished with few issues even in patients with poor baseline lung function. More centrally located SPNs may require completion lobectomy to achieve adequate margins. This can usually be achieved in patients with good lung function. Even in patients with poor lung function, a completion lobectomy may be possible due to the fact that, for most patients, the upper lobe has the least function and they may get a lung volume reduction surgery (LVRS)-type effect. This may require further testing with a quantitative Ventilation/Perfusion (V/Q) scan prior to return to the OR.

\section{Right middle lobe sublobar resection with close margin}

The right middle lobe presents several challenges due to its smaller size and two segments. First, any resection for a peripheral malignant SPN with close margin is likely to be a completion lobectomy due to small size of lobe. The patient and surgeon must be prepared preoperatively for this eventuality. Second, if the determination of close margin is made postoperatively, reresection surgery of the middle lobe will be adjacent to the hilum. After lymph node sampling of level 7 and 10 lymph nodes at initial surgery, this area will have significant inflammation or scarring depending on duration since original surgery (11). Third, the takeoff of the right middle lobe bronchus from the bronchus intermedius can make a completion lobectomy perilous and potentially risk the right lower lobe if great care is not taken during resection. A sleeve resection of the airway may be needed. For patients with poor lung function, additional resection should be considered carefully due to these risks.

\section{Right lower lobe sublobar resection with close margin}

The right lower lobe is the largest contributor to lung function due to its 5 segments. It often has less damage from smoking-related parenchymal destruction (12). For this lobe, if close margins are identified after resection of peripherally located malignant SPNs with close margins, either intraoperatively or after the original surgery, reresection is more feasible. Often a segmentectomy can be done rather than a completion lobectomy. Patients with poor lung function may even be candidates for sublobar resection for peripheral lesions.

Central malignant SPNs present a larger issue if they have close margins. Lesions located towards the diaphragm may be candidates for reresection postoperatively as there will be less scarring from hilar lymph node sampling farther from the hilum. However, lesions located closer to the hilum that didn't initially require lobectomy and need additional resection may mandate lower lobectomy intraoperatively. Through careful dissection due to hilar adhesions, bilobectomy can generally be avoided for these patients if they are taken back at a later date. Patients with poor baseline lung function and central lesions may not be able to withstand additional resection on an effort to obtain a better margin. These patients may require radiation or chemotherapy treatments to complete therapy for their 
malignant SPNs.

\section{Left upper lobe sublobar resection with close margin}

The left thoracic cavity presents unique challenges given how much of the chest is occupied by the heart, aorta, and origins of the carotid and subclavian arteries. For peripheral SPNs of the left upper lobe, if close margins are found intraoperatively, additional resection does not typically present an issue for patients with good lung function. If a close margin is found postoperatively for a peripheral lesion, additional resection can be done for patients with good lung function with the understanding a completion lobectomy may be necessary depending on the remaining parenchyma. Patients with poor lung function may be candidates for lobectomy on upper division segmentectomy due to worse function in the upper love for most patients with emphysema. Again, workup with quantitative V/Q scan can help determine this.

Central SPNs are more challenging for additional sublobar resection secondary to the proximity of the great vessels and the heart. Additional resection intraoperatively or on return to the $\mathrm{OR}$ will almost invariably require lobectomy if cancer has been found. Significant care must be used if return to the OR is delayed due to adhesions in the hilum. Patients with central SPNs and poor lung function should be counseled extensively on the risks of lobectomy at initial surgery and again for a planned return to the OR for an oncologic margin. Adjuvant therapy is worth considering in these cases with a multidisciplinary team.

\section{Left lower lobe sublobar resection with close margin}

Peripheral SPNs with close margins in the left lower lobe, like the right lower lobe, can typically undergo additional resection without completion lobectomy due to the large amount of parenchyma of the four lobar segments even for patients with poor lung function. When the close margin is identified during initial surgery, and especially if returning later to the OR, additional resection may entail lobectomy. Depending on the proposed degree of resection, adjuvant treatment may be advisable for malignant SPNs for patients with poor lung function. Even some patients with good lung function who have become marginal in performance may benefit from adjuvant therapy $v s$. resection.

\section{Conclusions}

While we have tried to present the preceding recommendations in a straightforward manner, the decision of what to do with a close margin for a malignant SPN is extraordinarily challenging. In general, peripheral SPNs in patients with good lung function can undergo additional resection when a close margin is discovered intraoperatively or on final pathology. Patients with poor lung function and peripheral SPNs need to be evaluated for possible lobectomy if additional resection is needed and this potential discussed with patients before their first surgery. Similarly, the possibility of lobectomy should be discussed at the outset for all patients with central SPNs regardless of lung function. Careful preoperative workup with echocardiogram to measure Pulmonary Artery pressure and a quantitative V/Q scan will aid in decision making.

\section{Acknowledgments}

Funding: None.

\section{Footnote}

Provenance and Peer Review: This article was commissioned by the editorial office, Current Challenges in Thoracic Surgery for the series "How to Evaluate, Diagnose and Treat Small Lung Nodules". The article did not undergo external peer review.

Conflicts of Interest: Both authors have completed the ICMJE uniform disclosure form (available at https://ccts. amegroups.com/article/view/10.21037/ccts-20-107/coif). The series "How to Evaluate, Diagnose and Treat Small Lung Nodules" was commissioned by the editorial office without any funding or sponsorship. DPD and SJS served as the unpaid Guest Editors of the series. SJS is a consultant for Ethicon and Covidien. The authors have no other conflicts of interest to declare.

Ethical Statement: The authors are accountable for all aspects of the work in ensuring that questions related to the accuracy or integrity of any part of the work are appropriately investigated and resolved.

Open Access Statement: This is an Open Access article distributed in accordance with the Creative Commons Attribution-NonCommercial-NoDerivs 4.0 International License (CC BY-NC-ND 4.0), which permits the non- 
commercial replication and distribution of the article with the strict proviso that no changes or edits are made and the original work is properly cited (including links to both the formal publication through the relevant DOI and the license). See: https://creativecommons.org/licenses/by-nc-nd/4.0/.

\section{References}

1. Hansell DM, Bankier AA, MacMahon H, et al. Fleischner Society: glossary of terms for thoracic imaging. Radiology 2008;246:697-722.

2. Aberle DR, DeMello S, Berg CD, et al. Results of the two incidence screenings in the National Lung Screening Trial. N Engl J Med 2013;369:920-31.

3. Mazzone PJ, Silvestri GA, Patel S, et al. Screening for Lung Cancer: CHEST Guideline and Expert Panel Report. Chest 2018;153:954-85.

4. National Comprehensive Cancer Network. Non-Small Cell Lung Cancer (Version 3.2020). Accessed May 5, 2020. Available online: https://www.nccn.org/professionals/ physician_gls/pdf/nscl.pdf

5. Sawabata N, Ohta M, Matsumura A, et al. Optimal distance of malignant negative margin in excision of nonsmall cell lung cancer: a multicenter prospective study. Ann Thorac Surg 2004;77:415-20.

6. Wolf AS, Swanson SJ, Yip R, et al. The Impact of Margins on Outcomes After Wedge Resection for Stage I Non-Small Cell Lung Cancer. Ann Thorac Surg

doi: $10.21037 /$ ccts-20-107

Cite this article as: Dolan DP, Swanson SJ. Solitary pulmonary nodules: what to do when the resection margin is too close. Curr Chall Thorac Surg 2021;3:32.
2017;104:1171-8.

7. Takahashi N, Sawabata N, Kawamura M, et al. Optimal sublobar resection for c-stage I non-small cell lung cancer: significance of margin distance to tumor size ratio and margin cytology (Supplementary analysis of KLSG-0801): complete republication. Gen Thorac Cardiovasc Surg 2019;67:690-6.

8. Mohiuddin K, Haneuse S, Sofer T, et al. Relationship between margin distance and local recurrence among patients undergoing wedge resection for small $(\leq 2 \mathrm{~cm})$ non-small cell lung cancer. J Thorac Cardiovasc Surg 2014;147:1169-75; discussion 1175-7.

9. Milano MT, Kong FS, Movsas B. Stereotactic body radiotherapy as salvage treatment for recurrence of nonsmall cell lung cancer after prior surgery or radiotherapy. Transl Lung Cancer Res 2019;8:78-87.

10. Kreuter M, Vansteenkiste J, Fischer JR, et al. Randomized phase 2 trial on refinement of early-stage NSCLC adjuvant chemotherapy with cisplatin and pemetrexed versus cisplatin and vinorelbine: the TREAT study. Ann Oncol 2013;24:986-992.

11. Rami-Porta, R. Staging Handbook in Thoracic Oncology. Second Edition. North Forty Myers: Editorial Rx Press, 2016.

12. Nemec SF, Bankier AA, Eisenberg RL. Upper lobepredominant diseases of the lung. AJR Am J Roentgenol 2013;200:W222-37. 